[white paper]

Diamond Open Access

\title{
O gato Toddy: acordado ou dormindo?
}

\author{
Colaboração Quântica Aberta ${ }^{1}$ \\ 19 de Setembro de 2022
}

\begin{abstract}
Resumo
Apresentamos uma adaptação do gato de Schroedinger tanto na forma de um circuito quântico como em sua contraparte analítica, com o objetivo de introduzir a computação quântica no ensino médio.
\end{abstract}

palavras-chave: superposição, circuitos e portas lógicas, computação quântica

A versão mais atualizada deste artigo está disponível em

https://osf .io/n5mp3/download

https://zenodo.org/record/5546102

Dedicamos este artigo à vida e ao gato Toddy, aquele que molhava sua patinha na água e, virando-a, trazia um pouco de água até sua boca, saciando sua sede de beber na fonte do conhecimento e da evolução.

${ }^{1}$ Todos os autores com suas afiliações aparecem no final deste artigo. 


\section{Preâmbulo}

1. Neste white paper [1,2], mostramos o princípio de superposição quântica por meio de uma adaptação do famigerado gato de Schroedinger [3], e transposto na forma de um Algoritmo de Resolução, seguindo a licença [4].

2. Um Algoritmo de Resolução é um material de cunho didáticopedagógico, que está entre a resolução completa e o gabarito de um problema.

\section{Introdução}

3. A principal vantagem da computação quântica (sobre a clássica) é que ela permite realizar uma quantidade colossal de cálculos de forma paralela.

\section{Notação (noções iniciais)}

4. := significa que o que está à esquerda é definido pelo que está à direita.

5. O símbolo | > é chamado ket.

6. $|\psi\rangle$ := função de onda (estado) do sistema quântico

7. $|0\rangle:=$ estado zero de um qubit

8. $|1\rangle:=$ estado um de um qubit

9. $|00\rangle,|01\rangle,|10\rangle,|11\rangle:=$ estados de um sistema com dois qubits

10. $|01\rangle:=$ estado zero do primeiro qubit e estado um do segundo qubit (note que cada posição dentro do ket representa uma partícula diferente) 


\section{Portas lógicas e circuitos quânticos}

11. Um circuito quântico é formado por uma ou mais portas lógicas quânticas.

12. Uma porta lógica atua em um objeto quântico, modificando seu estado.

13. Para o experimento proposto aqui, veremos apenas a porta quântica denominada CNOT.

\section{Qubit}

14. A palavra qubit vem do inglês quantum bit que, em português, significa bit quântico.

15. O qubit é um objeto quântico, podendo representar, por exemplo, a polarização de um fóton ou o spin do elétron.

16. O spin pode ser visto como a unidade mínima de carga elétrica circulante que produz campo magnético [5-7].

17. Basicamente, o que é importante lembrar acerca do qubit é que ele está em uma superposição quântica de dois estados.

18. Expressamos o qubit por meio da equação

$$
|\psi\rangle=a|0\rangle+b|1\rangle
$$

19. $a, b \in \mathbb{C} \operatorname{com}|a|^{2}+|b|^{2}=1$.

20. Note que $|a|^{2}$ é a probabilidade do sistema $|\psi\rangle$ colapsar no estado $|0\rangle$ e $|b|^{2}$ é a probabilidade de $|\psi\rangle$ colapsar em $|1\rangle$.

21. (19) significa que $a$ e $b$ são números complexos.

22. Os qubits são representados, também, por meio de matrizes. 
23.

$$
|0\rangle=\left(\begin{array}{l}
1 \\
0
\end{array}\right)
$$

24 .

$$
|1\rangle=\left(\begin{array}{l}
0 \\
1
\end{array}\right)
$$

\section{Dois qubits na forma matricial}

25. Lembre-se que $\left|q_{0} q_{1}\right\rangle=|01\rangle$ significa que o qubit $q_{0}$ está no estado $|0\rangle$, enquanto que o qubit $q_{1}$ está no estado $|1\rangle$.

26. Considere os seguintes estados de um sistema com dois qubits representados por meio de matrizes coluna.

27.

$$
|00\rangle=\left(\begin{array}{l}
1 \\
0 \\
0 \\
0
\end{array}\right)
$$

28.

$$
|01\rangle=\left(\begin{array}{l}
0 \\
1 \\
0 \\
0
\end{array}\right)
$$

29.

$$
|10\rangle=\left(\begin{array}{l}
0 \\
0 \\
1 \\
0
\end{array}\right)
$$


30.

$$
|11\rangle=\left(\begin{array}{l}
0 \\
0 \\
0 \\
1
\end{array}\right)
$$

\section{Porta quântica CNOT}

31. A porta quântica CNOT é representada pela matriz

$$
\mathrm{CNOT}=\left(\begin{array}{llll}
1 & 0 & 0 & 0 \\
0 & 1 & 0 & 0 \\
0 & 0 & 0 & 1 \\
0 & 0 & 1 & 0
\end{array}\right)
$$

32. A letra C vem de "controle".

33. Vamos ver, a seguir, como a porta lógica quântica CNOT altera os sistemas de dois qubits $|00\rangle,|01\rangle,|10\rangle$ e $|11\rangle$.

34. Vale ressaltar que a porta CNOT atua em um sistema com exatamente dois qubits. Por quê?

\section{CNOT $|00\rangle$}

35. Substituindo a matriz CNOT, (31), no sistema com dois qubits, (27),

$$
\operatorname{CNOT}|00\rangle=\left(\begin{array}{llll}
1 & 0 & 0 & 0 \\
0 & 1 & 0 & 0 \\
0 & 0 & 0 & 1 \\
0 & 0 & 1 & 0
\end{array}\right)\left(\begin{array}{l}
1 \\
0 \\
0 \\
0
\end{array}\right)=\left(\begin{array}{l}
1 \cdot 1+0 \cdot 0+0 \cdot 0+0 \cdot 0 \\
0 \cdot 1+1 \cdot 0+0 \cdot 0+0 \cdot 0 \\
0 \cdot 1+0 \cdot 0+0 \cdot 0+1 \cdot 0 \\
0 \cdot 1+0 \cdot 0+1 \cdot 0+0 \cdot 0
\end{array}\right)=\left(\begin{array}{l}
1 \\
0 \\
0 \\
0
\end{array}\right) \text {. }
$$

36. Assim,

$$
\text { CNOT }|00\rangle=|00\rangle \text {. }
$$




\section{CNOT $|01\rangle$}

37. Substitua a matriz do item (31) em (28) e mostre que

$$
\text { CNOT }|01\rangle=|01\rangle \text {. }
$$


CNOT $|10\rangle$

38. Substitua (31) em (29) e mostre que

$$
\mathrm{CNOT}|10\rangle=|11\rangle \text {. }
$$




\section{CNOT $|11\rangle$}

39. Substitua (31) em (30) e mostre que

$$
\mathrm{CNOT}|11\rangle=|10\rangle \text {. }
$$




\section{Interpretando os resultados}

40. De (36), (37), (38) e (39), temos os seguintes resultados:
(a) $\mathrm{CNOT}|00\rangle=|00\rangle$,
(b) $\mathrm{CNOT}|01\rangle=|01\rangle$,
(c) $\operatorname{CNOT}|10\rangle=|11\rangle$,
(d) $\operatorname{CNOT}|11\rangle=|10\rangle$.

41. O que a porta CNOT faz com o primeiro qubit em um sistema com dois qubits? E com o segundo? Qual é o qubit de controle e por quê? Explique detalhadamente. 


\section{Preparando o sistema}

42. Este é um experimento mental.

43. Suponha que um gato esteja em uma sala, conforme ilustrado na Fig. 1.

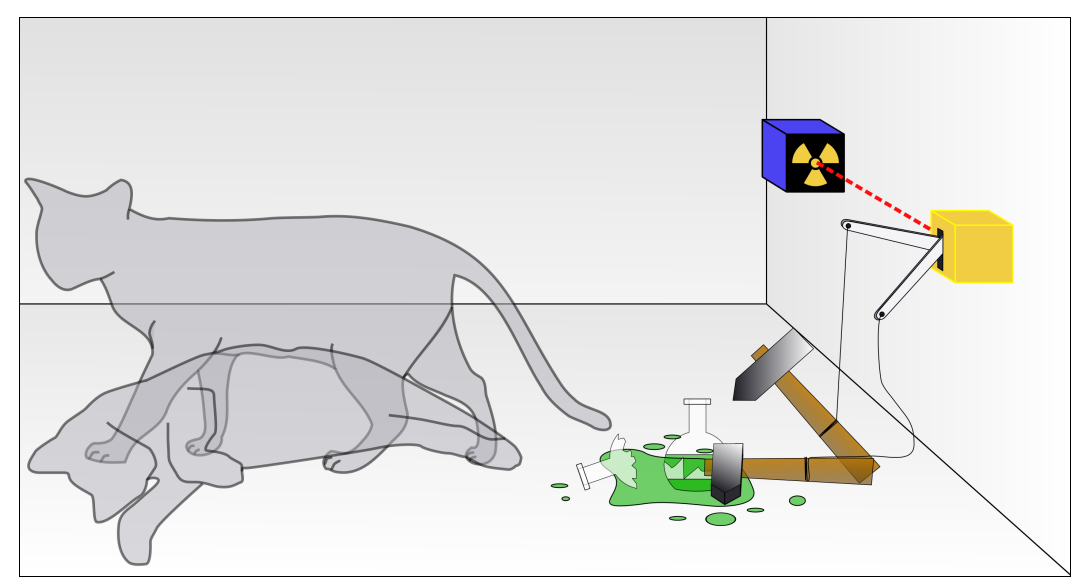

Figura 1: O gato Toddy está em uma superposição quântica dos estados acordado e dormindo. Trata-se de uma extrapolação clássica do chamado "problema da medida". Fonte da imagem: $[8,9]$.

44. Na parede, há uma caixa azul contendo um átomo que pode decair ou não.

45. Caso o átomo decaia, uma manivela é acionada, liberando um martelo, que quebra o vidro contendo um sonífero potente, que faz o gato dormir.

46. Caso o átomo não decaia, o gato permanece acordado.

\section{Matematizando o gato}

47. O experimento mental da seção anterior pode ser representado por meio de três qubits $\left(q_{0}, q_{1}\right.$ e $\left.q_{2}\right)$.

48. $q_{0}:=$ átomo decai ou não decai 
49. $q_{1}:=$ sonífero é liberado ou não é liberado

50. $q_{2}:=$ gato acordado ou dormindo

51. $|0\rangle:=$ o átomo não decai, o sonífero não é liberado, o gato está acordado

52. $|1\rangle:=$ o átomo decai, o sonífero é liberado, o gato está dormindo

53. De acordo com as definições apresentadas, considere os três qubits no seguinte estado

$$
\left|q_{0}\right\rangle=\left|q_{1}\right\rangle=\left|q_{2}\right\rangle=\frac{1}{\sqrt{2}}(|0\rangle+|1\rangle) .
$$

\section{$\mathrm{O}$ circuito}

54. O circuito quântico contendo o experimento mental descrito na seção anterior está ilustrado na Fig. 2.

55. A leitura do circuito é feita da esquerda para a direita.

56. No início (à esquerda) de cada linha horizontal está o input, isto é, o estado inicial de cada qubit.

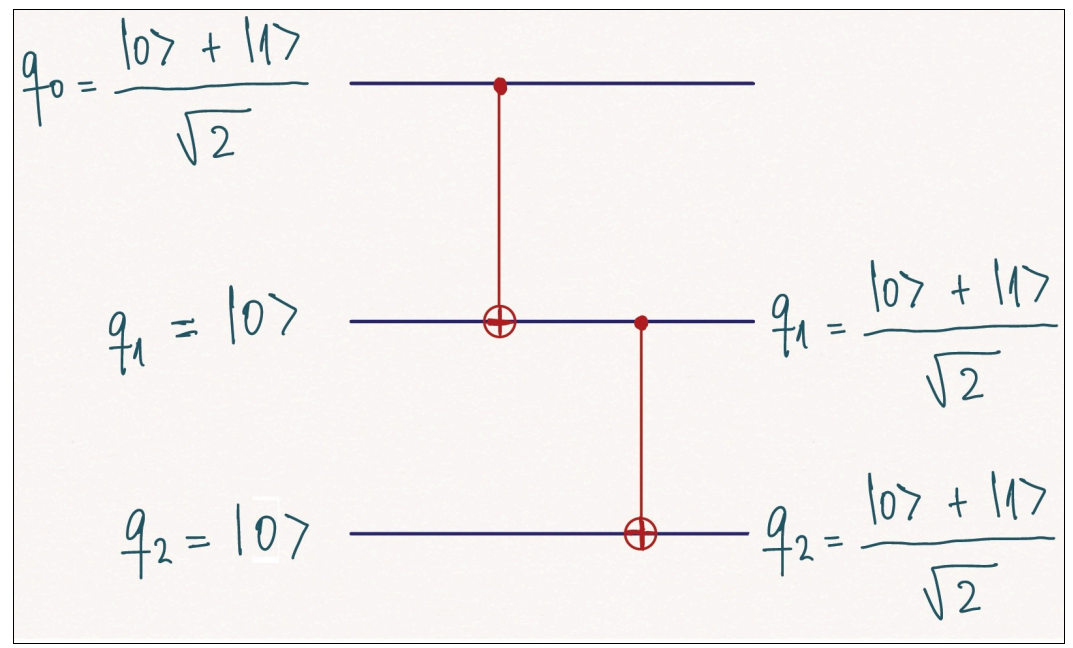

Figura 2: Sistema com três qubits que descreve a criação do gato Toddy.

57. As linhas horizontais representam os qubits $q_{0}, q_{1}$ e $q_{2}$. 
58. O símbolo contendo a linha vertical que conecta duas linhas horizontais significa a porta CNOT.

\section{Sistema com três qubits}

59. Note que a Fig. 2 descreve um sistema com três qubits.

60. A primeira porta CNOT está aplicada apenas nos qubits $q_{0}$ e $q_{1}$.

61. A segunda porta CNOT, por sua vez, está aplicada nos qubits $q_{1}$ e $q_{2}$.

62. Note que a segunda CNOT atua em $\left|q_{1}\right\rangle$ e $\left|q_{2}\right\rangle$ somente após a atuação da primeira porta CNOT.

63. Em equação, isso significa que o estado do sistema com três qubits da Fig. 2 é dado por

$$
|\psi\rangle=\operatorname{CNOT}_{q_{1} q_{2}}\left(\mathrm{CNOT}_{q_{0} q_{1}}\left|q_{0} q_{1}\right\rangle\right)\left|q_{2}\right\rangle .
$$

64. O estado inicial do átomo é dado por

$$
\left|q_{0}\right\rangle=\frac{1}{\sqrt{2}}(|0\rangle+|1\rangle),
$$

isto é, ele está em superposição de decaído e não decaído.

65. O estado inicial do sonífero é $\left|q_{1}\right\rangle=|0\rangle$ (não liberado).

66. O estado inicial do gato é $\left|q_{2}\right\rangle=|0\rangle$ (acordado).

\section{Resumindo a CNOT}

67. De (36), (37), (38) e (39), temos os seguintes resultados:

(a) $\operatorname{CNOT}|00\rangle=|00\rangle$,

(b) $\operatorname{CNOT}|01\rangle=|01\rangle$, 

(c) $\operatorname{CNOT}|10\rangle=|11\rangle$,
(d) $\operatorname{CNOT}|11\rangle=|10\rangle$.

68. Em palavras,

(i) se o primeiro qubit estiver no estado $|0\rangle$, a porta CNOT nãa altera o estado do segundo qubit;

(ii) se o primeiro qubit estiver no estado $|1\rangle$, a porta CNOT altera o estado do segundo qubit.

69. A porta CNOT atua nos estados $|00\rangle$ e $|01\rangle$ como uma matriz identidade.

\section{Calculando a porta CNOT nos qubits $q_{0}$ e $q_{1}$}

70. Nosso objetivo, nesse momento, é calcular $|\psi\rangle$ que aparece em (63).

71. Primeiro, calculamos $\mathrm{CNOT}_{q_{0} q_{1}}\left|q_{0} q_{1}\right\rangle$.

72. Substituindo, em (71), o estado inicial do átomo $\left|q_{0}\right\rangle=\frac{1}{\sqrt{2}}(|0\rangle+|1\rangle)$, dado em (64), e o estado inicial do sonífero, $\left|q_{1}\right\rangle=|0\rangle$, dado em (65),

$$
\operatorname{CNOT}_{q_{0} q_{1}}\left|q_{0} q_{1}\right\rangle=\frac{1}{\sqrt{2}} \operatorname{CNOT}(|0\rangle+|1\rangle)|0\rangle=\frac{1}{\sqrt{2}} \operatorname{CNOT}(|00\rangle+|10\rangle) .
$$

73.

$$
\operatorname{CNOT}_{q_{0} q_{1}}\left|q_{0} q_{1}\right\rangle=\frac{1}{\sqrt{2}} \mathrm{CNOT}|00\rangle+\frac{1}{\sqrt{2}} \mathrm{CNOT}|10\rangle .
$$

74. Utilizando (67) em (73),

$$
\operatorname{CNOT}_{q_{0} q_{1}}\left|q_{0} q_{1}\right\rangle=\frac{1}{\sqrt{2}}|00\rangle+\frac{1}{\sqrt{2}}|11\rangle .
$$




\section{Calculando a porta CNOT nos qubits $q_{1}$ e $q_{2}$ (resultado da seção anterior)}

75. O próximo passo é substituir (74) em (63). 76.

$$
|\psi\rangle=\frac{1}{\sqrt{2}} \operatorname{CNOT}_{q_{1} q_{2}}(|00\rangle+|11\rangle)\left|q_{2}\right\rangle .
$$

77. Substituindo, em (76), o estado inicial do gato, $\left|q_{2}\right\rangle=|0\rangle$, dado em (66),

$$
|\psi\rangle=\frac{1}{\sqrt{2}} \operatorname{CNOT}_{q_{1} q_{2}}(|00\rangle+|11\rangle)|0\rangle .
$$

78. Fazendo a distributiva do produto tensorial em (77),

$$
|\psi\rangle=\frac{1}{\sqrt{2}} \operatorname{CNOT}_{q_{1} q_{2}}(|000\rangle+|110\rangle) .
$$

79. Como $\mathrm{CNOT}_{q_{1} q_{2}}$ só atua nos qubits $\left|q_{1}\right\rangle$ e $\left|q_{2}\right\rangle$, temos que

$$
|\psi\rangle=\frac{1}{\sqrt{2}}(|0\rangle \text { CNOT }|00\rangle+|1\rangle \text { CNOT }|10\rangle) .
$$

80. Utilize (67) em (79) e mostre que

$$
|\psi\rangle=\frac{1}{\sqrt{2}}(|000\rangle+|111\rangle) .
$$




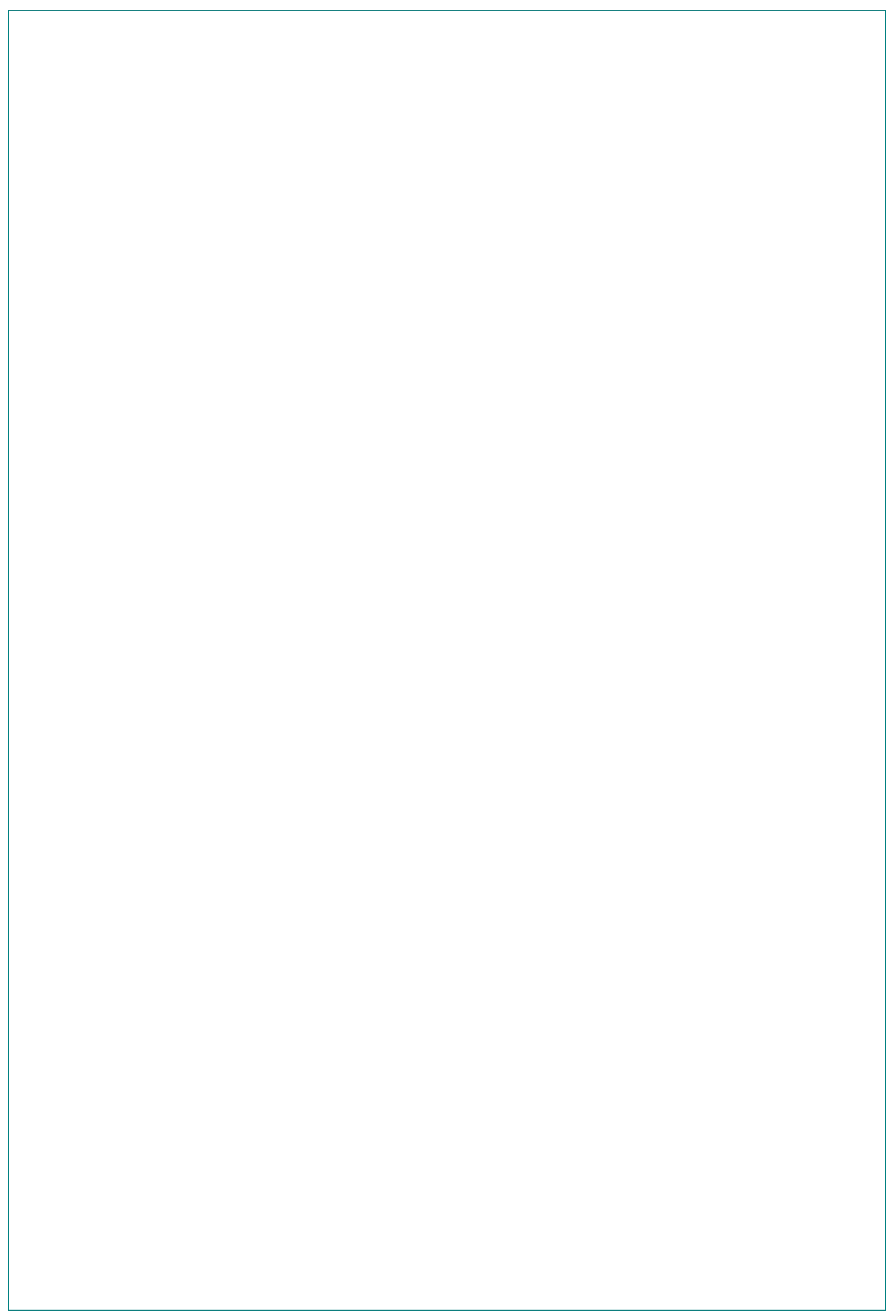




\section{Qual é o significado de $|\psi\rangle$ ?}

81. Em resumo, mostramos que

$$
|\psi\rangle=\frac{1}{\sqrt{2}}(|000\rangle+|111\rangle) .
$$

82. A equação em (81) representa a superposição quântica (via emaranhamento) entre o átomo $\left(q_{0}\right)$, a alavanca $\left(q_{1}\right)$ e o sonífero $\left(q_{2}\right)$.

83. O emaranhamento é um caso particular de superposição quântica.

84. Lembre-se que o ket em (81) está escrito na forma $\left|q_{0} q_{1} q_{2}\right\rangle$, sendo que a primeira posição é referente ao qubit $q_{0}$, a segunda referente a $q_{1} \mathrm{e}$ a terceira, a $q_{2}$.

85. Então, temos os seguintes resultados.

86. $|000\rangle:=$ estado em que o átomo $\left(q_{0}\right)$ não decai, a alavanca não é acionada $\left(q_{1}\right)$ e o gato está acordado $\left(q_{2}\right)$.

87. $|111\rangle:=$ estado em que o átomo $\left(q_{0}\right)$ decai, a alavanca é acionada $\left(q_{1}\right)$ e o gato está dormindo $\left(q_{2}\right)$.

88. Para saber se o gato está acordado ou dormindo, é preciso abrir a porta que, fisicamente, significa medir o sistema.

89. Se o resultado da medição mostrar $\left|q_{0}\right\rangle=|0\rangle$, então temos a situação descrita em (86).

90. Se o resultado da medição mostrar $\left|q_{0}\right\rangle=|1\rangle$, então temos a situação (87).

\section{Considerações Finais}

91. Calculamos as transformações da porta quântica CNOT em um sistema com três qubits para simular o gato Toddy, uma adaptação do gato de Schroedinger em uma versão mais saudável. 


\section{Ciência Aberta}

O arquivo latex para este artigo, juntamente com outros arquivos suplementares, estão disponíveis em [10]. Seja coautor(a) deste artigo, envie sua contribuição para mplobo@uft. edu.br.

\section{Consentimento}

Os autores concordam com [11].

\section{Como citar este artigo?}

https://doi.org/10.31219/osf .io/n5mp3

https://zenodo.org/record/5546102

\section{Licença}

CC-By Attribution 4.0 International [4]

\section{Referências}

[1] Lobo, Matheus P. "Uma Revista Aberta." OSF Preprints, 19 July 2021. https://doi.org/10.31219/osf.io/fjb9a

[2] Lobo, Matheus P. "Microarticles." OSF Preprints, 28 Oct. 2019. https://doi.org/10.31219/osf.io/ejrct

[3] Sutor, Robert S. Dancing with Qubits: How quantum computing works and how it can change the world. Packt Publishing Ltd, 2019.

[4] CC. Creative Commons. CC-By Attribution 4.0 International. https://creativecommons.org/licenses/by/4.0 
[5] Ohanian, Hans C. "What is spin?." American Journal of Physics 54.6 (1986): 500-505.

[6] Lobo, Matheus P. "Spin Is a Circulating Field." OSF Preprints, 30 June 2019. https://doi .org/10.31219/osf .io/khnpj

[7] Lobo, Matheus P. "Spin Is a Superposition of Circular Charged Trajectories." OSF Preprints, 30 June 2019.

https://doi.org/10.31219/osf.io/tv2rb

[8] Wikipedia. Schrödinger's cat.

https://en.wikipedia.org/wiki/Schr\%C3\%B6dinger\%27s_cat\#/ media/File:Schrodingers_cat.svg

[9] CC. Creative Commons. Attribution-ShareAlike 3.0 Unported (CC BY-SA 3.0).

https://creativecommons.org/licenses/by-sa/3.0

[10] Lobo, Matheus P. "Open Journal of Mathematics and Physics (OJMP)." OSF, 21 Apr. 2020.

https://doi.org/10.17605/osf .io/6hzyp

[11] Lobo, Matheus P. "Simple Guidelines for Authors: Open Journal of Mathematics and Physics." OSF Preprints, 15 Nov. 2019.

https://doi.org/10.31219/osf .io/fk836 


\title{
Colaboração Quântica Aberta
}

Matheus Pereira Lobo (autor principal, mplobo@uft.edu.br) ) $^{1,2,3}$ https://orcid.org/0000-0003-4554-1372

\section{Alex Kevyn dos Anjos Carreiro ${ }^{1,4}$ Maurício Ribeiro Gonçalves ${ }^{1,5}$}

\author{
${ }^{1}$ Universidade Federal do Tocantins (Brasil) \\ ${ }^{2}$ Universidade Federal do Norte do Tocantins (Brasil) \\ ${ }^{3}$ Universidade Aberta (UAb, Portugal) \\ ${ }^{4}$ Escola Estadual Francisco Máximo de Sousa (TO, Brasil) \\ ${ }^{5}$ Centro de Ensino Parsondas de Carvalho (MA, Brasil)
}




\section{APÊNDICE}

\section{Simplificando o gato Toddy}

92. A seguir, apresentamos um sistema alternativo para a criação da superposição quântica utilizando-se apenas dois qubits.

93. $q_{0}:=$ átomo decai ou não decai

94. $q_{1}:=$ "sonífero é liberado (e o gato dorme)" ou "não é liberado (e o gato permanece acordado)"

95. $|0\rangle:=$ o átomo não decai, o sonífero não é liberado e o gato está acordado

96. $|1\rangle:=$ o átomo decai, o sonífero é liberado e o gato está dormindo

97. De forma similar, considere os dois qubits no seguinte estado

$$
\left|q_{0}\right\rangle=\left|q_{1}\right\rangle=\frac{1}{\sqrt{2}}(|0\rangle+|1\rangle) .
$$

98. Conforme calculado anteriormente, temos que

$$
\text { CNOT }\left|q_{0} q_{1}\right\rangle=\frac{1}{\sqrt{2}}(|00\rangle+|11\rangle) \text {. }
$$

99. Assim, ao medir se o frasco está quebrado ou não, conseguimos inferir o estado do gato. 